\title{
Applying Root-Locus Techniques to the Analysis of Coupled Modes in Piano Strings
}

\author{
Timothy S. Stilson \\ Center for Computer Research in Music and Acoustics, Stanford University \\ stilti@ccrma.stanford.edu, http://www-ccrma.stanford.edu/ ${ }^{\sim}$ stilti
}

\begin{abstract}
Previous work in the study of coupled piano string behavior has focused analytically on the interaction of a pair of coupled modes, noting that the rest of the string modes couple similarly. In this paper, multi-string coupling is analyzed with the root-locus method, which describes the movement of system poles under variations in a single parameter, such as coupling magnitude. Many effects, for example, two-stage decay, can be understood in terms of system pole location. Often, an intuitive understanding of pole movement under variations in a parameter, such as a single mode frequency or coupling coefficient, can be developed. Three-mode coupling is explored and interpreted, as is variation in coupling behavior at different string harmonics.
\end{abstract}

\section{Introduction}

Previous work in the study of coupled piano string behavior ([1], [2], [3]) has either focused analytically on the interaction of a pair of coupled modes, noting that the rest of the string modes couple similarly, or has studied the coupling experimentally. The equations describing threestring coupling (along the lines of [3]) become extremely complex and one can easily become lost in interpreting them. In this paper, we will show how one can analyze the coupling behavior in terms of the Root Locus analysis method, which analyzes the location of the poles of a closed-loop linear system according to the pole and zeros of the open-loop system and under variation of the feedback gain. Root Loci were for decades drawn by hand, so that a lore was developed about patterns that appear in root loci; this lore represents an intuition that can be acquired and applied to get a feel for the behaviors of closed-loop systems - coupled strings, in this case.

The coupling of two and three modes, as occurs in sets of unison piano strings, is studied, along with the coupling behavior of multiple groups of modes, such as the multiple harmonics of the strings. Many effects, for example, beating and two-stage decay, can be understood in terms of the system pole locations (since for impulsively driven systems such as pianos, the normal behavior of the system is given by the impulse response of the system), which makes the root-loci directly interpretable. Furthermore, rather complex, frequency-dependent coupling impedances can easily be included in the analysis, allowing an exploration of the variation in coupling behavior at different string harmonics.

\section{Root Locus Analysis}

This technique, which originated in the analysis of linear feedback control systems, analyzed the poles of a closedloop feedback system in terms of the open-loop transfer function and the (variable) loop gain. Textbooks on clas-

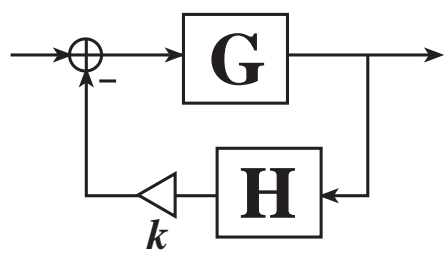

Figure 1: Linear system in Root-Locus Form

sical control systems, such as [4], provide rules on drawing root-loci. The most important rule to note is that the closed-loop poles coincide with the open-loop poles when $k=0$, and move to coincide with the open-loop zeros as $k \rightarrow \infty$. Thus we gather quite a bit of information simply by plotting the open loop poles and zeros. Another rule is that the paths of the poles are given by the equation $\angle(G H)=\pi$. We can use this rule to draw root-loci of non-rational linear systems, which show up in the analysis of continuous-time string coupling.

\section{Coupled Modes}

Let $G_{\text {Forward }}=\frac{1}{s-p_{1}}+\frac{1}{s-p_{2}}$, and let $G_{\text {load }}=e^{j \phi}$. We can then interpret $k$ as the magnitude of the coupling between the two open-loop modes $p_{1}$ and $p_{2}$. 


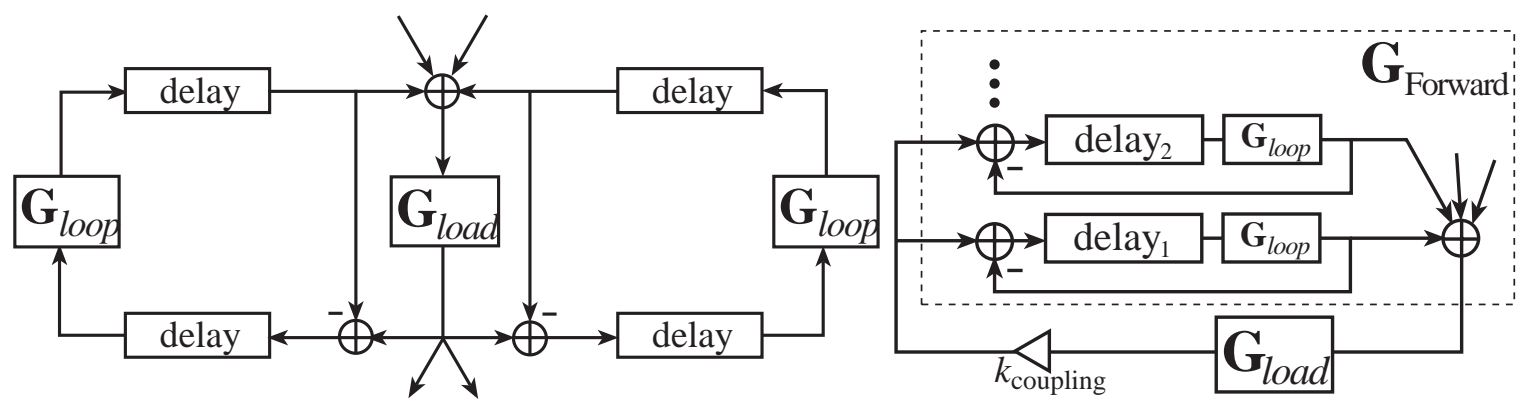

Figure 2: Waveguide representation of $N$ coupled strings [5], and rearranged into Root-Locus form
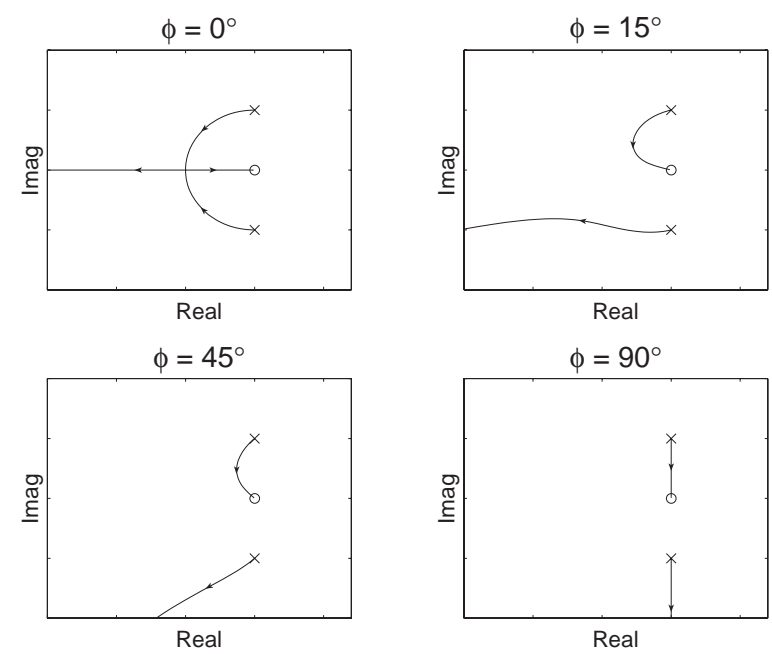

Figure 3: Sample 2-Coupled-Mode Loci

We draw root-loci for $k>0$, and for different values of $\phi$ (Figure 3). For small coupling (small $k$ ), the poles stay near the open-loop poles. The impulse response of the system in this case would have betaing between the modes. As the coupling gets stronger, the poles move into a region where one pole has a faster decay than the other, giving the well-known two-stage decay. In this region, the effect of the coupling angle is to "rotate" the root locus, which detunes the modes when they are in the two-stage region. This detuning accounts for the momentary dip of the decay at the crossover between stages ([1], Figure 11, or [3], Figure $6 \mathrm{~b}$ ). The rotation of the locus also explains the "mode repulsion" effect for reactive impedances (lowerright plot: the 90-degree coupling case).

It is important to note that the shapes of the loci are relatively independent of the actual mistuning of the modes. These loci will look the same for any vertical spacing of the two modes. The distance between the open-loop poles does affect the range of $k$ over which different behaviors will occur: the closed loop poles land at the intersections of the $\angle(G H)=\pi$ and $|G H|=1 / k$ contours; when the poles are closer together, the magnitude of $G H$ is larger in the region where the root-locus has interesting behavior
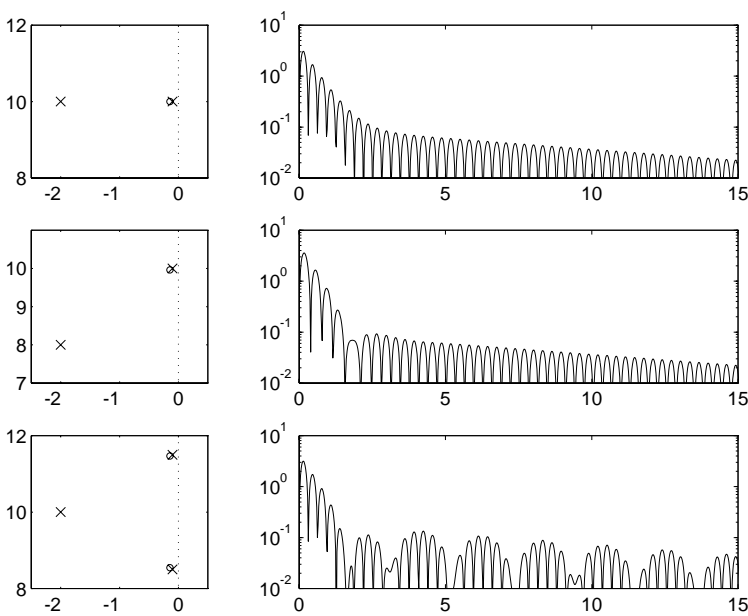

Figure 4: Rectified impulse responses for various twostage closed-loop pole configurations

(like where the locus tracks intersect in the real-coupling case). The main result of this is that one can also move the system between beating and two-stage decay by fixing $k$ and adjusting the mistuning of the modes (which is how Weinreich did it in [1]).

\section{Coupled Strings}

Referencing Figure 2, we can set up the root-locus for a system of coupled strings by letting:

$$
G_{\mathrm{Forward}}=\sum_{i=1}^{N} \frac{-G_{\text {loop }} e^{-s T_{i}}}{1-G_{\text {loop }_{i}} e^{-s T_{i}}}
$$

Where the $T_{i}$ are the round-trip delays of each string, and $G_{\text {loop }_{i}}$ are the lumped round-trip loss of the strings. We can draw the root locus vs. $k_{\text {coupling }}$ of the coupled system by evaluating the contour $\angle\left(G_{\text {Forward }}(s) G_{\text {load }}(s)\right)=0$ in the s-plane. ${ }^{1}$ We can determine the closed-loop pole

\footnotetext{
${ }^{1}$ We evaluate at $L(\bullet)=0$ instead of $L(\bullet)=\pi$ because the system is missing the extra sign inversion in the loop that the system in Figure 1 has due to the subraction in the loop.
} 
locations for a given $k_{\text {coupling }}$ by evaluating the contour $\left|G_{\text {Forward }}(s) G_{\text {load }}(s)\right|=1 / k_{\text {coupling }}$ and locating its intersections with the root locus.
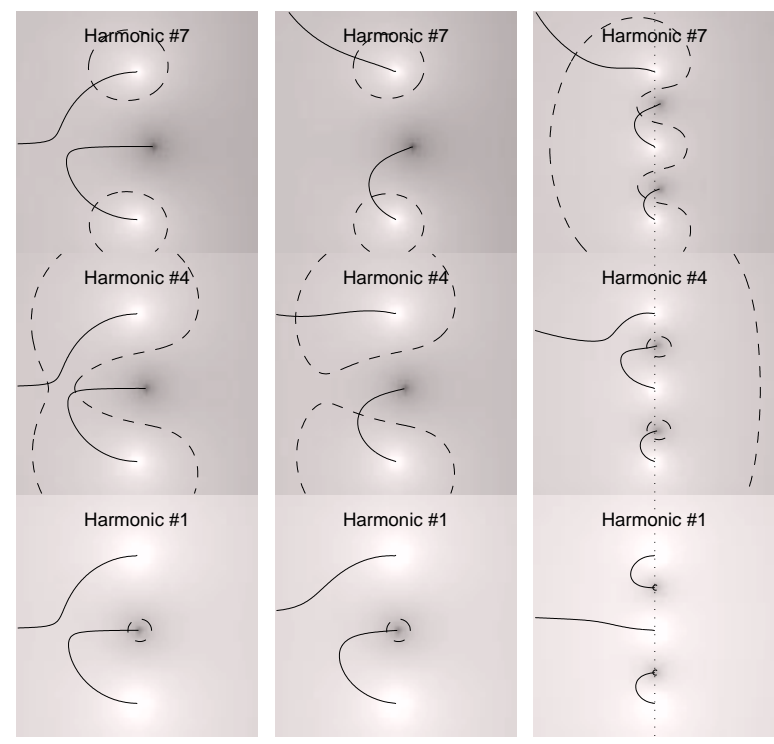

Figure 5: String Loci, left: two strings, real coupling, middle: two strings, one-pole $G_{l o a d}$, right: three strings, onepole $G_{\text {load }}$.

In Figure 5, we have plotted select sections of the root loci for three different systems. In the left system, we see three harmonics for the case of two-string coupling with a real scalar $G_{\text {load }}$. This case shows the fact that the distance between the poles will change the 'effective' coupling strength: the low harmonics, being closer, are strongly into two-stage decay, with the fast mode out of the picture to the left; whereas at the higher harmonics, the detuned open-loop poles end up further apart, so that at the seventh harmonic, the poles are still in a beating configuration. This emphasizes that different harmonics will couple differently with the same coupling constant, simply due to their pole spacing.

The middle case has $G_{\text {load }}$ set to a onepole lowpass filter $\frac{a}{s+a}$. This coupling is now frequency dependant, so that each harmonic will see a different coupling factor. Since the phase of the onepole filter approaches -90 degrees at high frequencies, we would expect that higher harmonics will display coupling patterns that are more and more rotated, along with the distance-induced reduction in coupling, which can be seen in the figure.

The right-hand case has three strings, along with the one-pole frequency dependent coupling. An important effect can be seen at the first harmonic, where the coupling is nearly real: the sum of the three modes has two zeros "inbetween" the poles, this causes two of the poles to stay at slow decay when the system is strongly coupled, so that only the third pole goes into fast decay. Thus, during the second stage of decay, the two slow modes will beat with each other, an effect that is seen in [2] and [3].

\section{Conclusions}

The Root Locus visualizes the process of mode coupling in systems, and can provide intuition on the coupling behavior. Some important facts about multi-string coupling are deduced from the root loci:

- In 3-mode coupling, two modes stay at slow decays, this gives the beating in the second stage

- Different string harmonics couple differently:

- Higher harmonics are more detuned (absolute detuning, not relative), this makes the higher harmonics "less coupled"

- Frequency-dependent coupling causes each harmonic to couple differently, for example each harmonic couples with a different coupling phase angle.

\section{References}

[1] Weinreich, G. 1977. "Coupled Piano Strings", J. Acoust. Soc. Am. Vol. 62, No. 6, pp. 1474-84.

[2] Hundley, C. et al 1978. "Factors Contributing to the Multiple Rate of Piano Tone Decay", J. Acoust. Soc. Am. Vol. 64, No. 4, pp. 1303-09.

[3] Nakamura, I. 1989. "Fundamental Theory and Computer simulation of the Decay Characteristics of Piano Sound", J. Acoust. Soc. Jpn. Vol. 10, No. 5, pp. 289-97.

[4] Franklin, G., Powell, J.D., Emami-Naeini, A. 1994. Feedback Control of Dynamic Systems, New York: Addison-Wesley, pp. 243-336.

[5] Smith, J.O. 1993. "Efficient Synthesis of Stringed Musical Instruments”, ICMC93, Tokyo, pp.64-71.

The annotated viewgraphs of a talk on this topic, including a more tutorial discussion and more examples, is available at the web site (see the author line) at http://../ ${ }^{\sim}$ stilti/papers/RLTalk2.pdf 